\title{
Knowledge database and case-based reasoning
}

\author{
W. Raskob, S. Möhrle and S. Bai \\ KIT - Karlsruhe Institute of Technology, Hermann-von-Helmholtz-Platz 1, 76344 Eggenstein-Leopoldshafen, Germany.
}

\begin{abstract}
Nuclear emergencies are characterised by severe disruptions in society's functionality and adverse impacts on human, environment and economy. Decision-making in times of such crises is complex and usually accompanied by acute time pressure. Environment can change rapidly and decisions may have to be made based on uncertain information. IT-based decision support can systematically help to identify response and recovery measures, especially when time for decision-making is sparse, when numerous options exist or when events are not completely anticipated. This paper reviews the case- and scenario-based approach implemented in the so-called analytical platform to support the management of nuclear events in different accident phases. Important information needed for decision-making as well as approaches to reusing experience from previous events and the fictitious scenarios calculated by Java version of the Real-time On-line Decision Support System are discussed. Suitable management options based on similar historic events and scenarios might be identified to support disaster management.
\end{abstract}

Keywords: knowledge database / case-based reasoning / analytical platform / decision support / PREPARE

\section{Introduction}

The European project PREPARE (innovative integrated tools and platforms for radiological emergency preparedness and post-accident response in Europe) aims at closing gaps that have been identified in nuclear and radiological preparedness following the first evaluation of the Fukushima disaster. In this project, the methodological approaches, the knowledge database and case-based reasoning (CBR) are part of the socalled analytical platform, to improve information collection and exchange during such types of disasters.

CBR is a problem-solving methodology, which utilises knowledge from previously experienced problematic situations (Aamodt and Plaza, 1994). The knowledge is comprised of previous cases, knowledge representation, similarity measures and the adaptation mechanism (Richter, 1995). CBR can be described as a four Rs cycle (Figure 1). The first step is to retrieve the case which needs to identify, assess and describe one problem. And then, similar cases would be determined from one case base in order to reuse the solutions. The third step is to revise the case which would evaluate the application of the proposed solutions and adapt them to the current circumstances, if necessary. Finally, the case base would be retained, in other words, updated by storing the new case with its possibly corrected or improved and confirmed solutions.

The small number of historic events is a limitation for CBR. Therefore, scenarios, confirmed by experts, are used as extension of the case bases. They support the discussions about possible event developments. Such an application of knowledge gained from historic events or the calculation of fictitious scenarios by the Java version of the Real-time On-line Decision Support System (JRODOS) (Raskob et al., 2011) was firstly introduced (Moehrle and Raskob, 2015) especially in nuclear and radiological emergency management and preparedness.

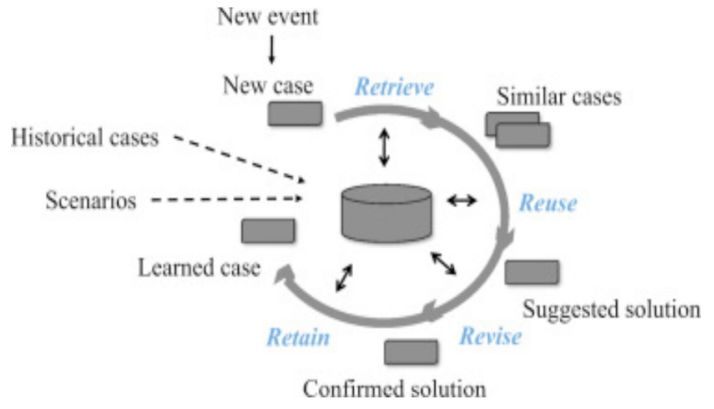

Figure 1. Case-based reasoning (Aamodt and Plaza, 1994).

\section{Knowledge database}

Decision makers use their knowledge to make decisions, e.g. they use cases from the past. This is replicated with our knowledge database. To characterise a scenario/event, attributes related to events are elucidated and structured. One event can be subdivided into affected areas and accident phases that account for differences in the status of the release, type and urgency of countermeasures, type and availability of resources and relevance of exposure pathways (Figure 2). Experts of different institutions in Europe voted on the attributes concerning their importance for specific phases ('threat phase', 'release phase', 'transitional phase' and 'long-term post-accident phase').

\subsection{Historic cases}

A total of 16 historic cases, from the Windscale fire, Chernobyl and Fukushima accidents and the poisoning of Alexander Litvinenko, are in the database. In case of Chernobyl, five affected areas were taken into account. For 


\begin{tabular}{|c|c|c|c|}
\hline \multicolumn{2}{|c|}{ During the incident } & \multicolumn{2}{|r|}{ After the releases } \\
\hline \multicolumn{2}{|c|}{ Urgency phase } & \multicolumn{2}{|c|}{ Post-accident phase } \\
\hline Pre-release phase & Release phase & Transition phase & Long-term post-accident phase \\
\hline \multicolumn{2}{|c|}{ Hours/days } & Days/weeks/months & Weeks/months/years/decades \\
\hline \multicolumn{2}{|c|}{ Early protective actions } & $\begin{array}{l}\text { Check/revoke } \\
\text { early actions } \\
\text { Preparation of } \\
\text { long-term actions }\end{array}$ & $\begin{array}{l}\text { Objective: Rehabilitation of } \\
\text { living conditions }\end{array}$ \\
\hline
\end{tabular}

Figure 2. Phases of a nuclear accident (German Commission on Radiological Protection, 2014).

the Windscale fire, a ban of milk is considered. And in the event of the poisoning of Alexander Litvinenko, the amount of people with urine tests in UK and other countries were stored. More details can be found in the paper (Moehrle and Raskob, 2015).

\subsection{Fictitious scenarios}

\subsubsection{Pre-release phase}

A total of 10 cases are introduced by the HERCAWENRA scheme which proposes a methodology for a common European approach to recommend urgent protective actions as well as a minimum common level of preparation for the corresponding actions (Herca and Wenra, 2014).

- Evacuation should be prepared up to $5 \mathrm{~km}$ around nuclear power plants and sheltering and iodine thyroid blocking (ITB) up to $20 \mathrm{~km}$.

- A general strategy should be defined in order to extend evacuation up to $20 \mathrm{~km}$ and sheltering and ITB up to $100 \mathrm{~km}$.

The corresponding parameters are 'risk of a core melt', 'integrity of the containment', 'wind direction known' and 'evacuation performed in time or not'.

\subsubsection{Early phase (release phase)}

A total of 96 fictitious scenarios have been prepared with JRODOS. To facilitate the integration into the database, a procedure has been developed automatically extracting the important information of the database at least in the early phases. For the later phase, information still has to be transferred manually to the knowledge database. The key characteristics of a scenario are 'INES scale' (5, 6 and 7), 'season', 'weather' (dry, with/without rain and low/medium/ stable wind speeds) and 'population distribution' (urban, rural and metropolitan). Main output parameters are the 'size of evacuation/sheltering area and the number of affected people', 'size of distribution area of iodine tablets for adults/children and the number of affected people', 'cost' and 'average shelter factor'. The regression analysis of the scenarios reveals the 'INES scale' as most important, followed by 'weather', while 'season' is of nearly no importance for the early phase actions.

\subsubsection{Long-term scenarios}

A total of 8 long-term scenarios corresponding to the season, weather conditions and the population distribution have been added into the database. All the data were normalised to a certain contamination level $(100 \mathrm{mSv}$ in the first year) as well as the area of one square kilometre. This allows for scaling to other conditions. More scenarios will be defined in the future. The result parameters are 'strategies' (effective and less effective), 'management options and targets', 'order of implementation', 'cost', 'waste' and 'dose reduction factor'.

\section{Conclusions}

By using the CBR application, one event can be described and similar cases can be retrieved from the historic events and fictitious scenarios. When using the analytical platform, the similar cases as well as their strategies, in particular their consequences, are available in the tool 'historic analysis'. This allows facilitating the selection of suitable strategies in particular under uncertainty. So far, the database contains 130 cases including 16 historic ones, 10 cases for the pre-release phase, 96 scenarios for the release phase and 8 cases for the long-term phase.

In the near future, more scenarios for the long-term phase will be developed and also non-nuclear events will be integrated.

Acknowledgement. The research leading to these results has received funding from the European Atomic Energy Community Seventh Framework Programme FP7/2012-?2013 under grant agreement 323287.

\section{References}

Aamodt A., Plaza E. (1994) Case-based reasoning: foundational issues, methodological variations, and system approaches, $A I$ Commun. 7, 39-59.

German Commission on Radiological Protection (2014) Basic radiological principles for decisions on measures for the protection of the population against incidents involving releases of radionuclides [WWW Document]. URL: http://www.ssk.de/Share dDocs/Beratungsergebnisse_PDF/2014/RadiologischeGrundla gen_e.pdf?_blob=publicationFile (accessed 01.05.15).

Herca, Wenra (2014) HERCA-WENRA Approach for a Better Cross-Border Coordination of Protective Actions during the Early Phase of a Nuclear Accident.

Moehrle S., Raskob W. (2015) Structuring and reusing knowledge from historical events for supporting nuclear emergency and remediation management, Eng. Appl. Artif. Intell. 46, 303-311.

Raskob W., Trybushnyi D., Ievdin I., Zheleznyak M. (2011) JRODOS: platform for improved long term countermeasures modelling and management, Radioprotection 46, S731-S736.

Richter M.M. (1995) The knowledge contained in similarity measures. In: Invited talk at the first international conference on CBR (ICCBR-95).

Cite this article as: W. Raskob, S. Möhrle, S. Bai. Knowledge database and case-based reasoning. Radioprotection 51(HS2), S185-S186 (2016). 